\title{
LOS DEBERES ESCOLARES EN EL MARCO DE LAS RELACIONES FAMILIA -ESCUELA
}

\section{HOMEWORK IN THE CONTEXT OF FAMILY-SCHOOL RELATIONSHIPS}

\author{
María del Luján González Tornaría \\ Albertina Guerra \\ Sofía Prato \\ Patricia Barrera \\ Universidad Católica del Uruguay, Uruguay
}

\begin{abstract}
Resumen: Esta comunicación presenta la primera etapa de una investigación que se centra en los deberes escolares y su influencia en la relación entre las familias y los centros educativos. Se ofrece una reseña actualizada acerca de la literatura internacional sobre el tema y el diseño de la investigación internacional de la cual este estudio forma parte. Por último, se da cuenta de la etapa de estudio piloto de los instrumentos de investigación en idioma español, que se está empezando a transitar.
\end{abstract}

Palabras clave: relación familia-escuela, tareas domiciliarias, familia, educación.

\begin{abstract}
This communication introduces the first stage of an investigation that focuses on homework and its influence on the relationship between families and schools (educational centers). An updated international literature review on the subject is provided. Furthermore, the design of the international investigation (which comprises the present study) is itemized. Finally, authors notify the stage of instrument's pilot study in Spanish language.
\end{abstract}

Keywords: family school relationship, howmork, family, education.

En la investigación realizada en Uruguay colaboró, en la etapa de diseño y recolección de datos la Lic. Natalia Cuesta. En cuanto a los aspectos metodológicos la colaboración fue brindada por el Lic.Prof. Daniel Costa y el Prof. Daniel Sucazes.

\section{LOS DEBERES ESCOLARES EN LA MIRA}

Los deberes escolares constituyen la parte emergente del trabajo escolar para los padres. Ellos pueden constituir una instancia de intercambio privilegiado con la escuela, y por ello, los padres deben jugar un rol fundamental en los mismos (Cooper, Lindsay y Nye, 2000; Hoover-Dempsey et al, 2001). Al punto que Epstein (1988) incluye el involucramiento de los padres en las tareas, como una de sus posibles formas de participación, junto con el ejercicio de responsabilidades básicas tales como la implicancia directa de los padres en la escuela y la capacidad de tomar decisiones en diferentes instancias educativas.
Los deberes constituyen una herramienta que ayuda a los maestros a demostrar lo trabajado en clase, y a los padres a seguir la evolución de sus hijos.

Adoptado como mecanismo de control, es posible que el deber cumpla con el rol de satisfacer la expectativa de los adultos involucrados. De este modo, los padres esperan que un buen docente mande tareas, mientas que los docentes asumen esta actividad como obligatoria dentro de su rol. Los deberes pierden su objetivo cuando no se ajustan a las consignas programáticas establecidas porque estas no son precisas. En esta línea la literatura llama a una revisión consciente y honesta del por qué y para qué de las tareas domiciliarias. Justamente, Cooper (2003, en Deslandes 2009 a) 
sostiene que los deberes si no son adecuadamente asignados y monitoreados pueden tener efectos negativos.

Por su lado, a medida que crecen, los niños van generando "inmunidad" frente a la tarea. Esto es, están menos interesados en estudiar, hacer deberes e investigar. Si bien actualmente pueden estar en contacto con mucha información, a veces no están suficientemente motivados para comprenderla e integrarla (Jackson, 2007). Los niños generalmente realizan los deberes con propósitos diferentes o menos relevantes a los de un adulto. Según Bempechat (2004) los padres y profesores comparten por lo general, la misma visión en cuanto al objetivo de los deberes y lecciones. Estos serían: reforzar lo aprendido en clase, desarrollar conductas autorregulatorias, practicar y reforzar los contenidos tratados en clase.

El tema de las tareas domiciliarias, si bien tiene larga data, es fuente de conflicto entre familias y escuelas. Este hecho ha motivado que en algunos países los Ministerios de Educación se hayan pronunciado al respecto, tratando de regular el tema. Este es el caso del Ministerio de Educación de Québec que ha puesto, desde el año 2003, medidas de ayuda para los deberes en las escuelas primarias.

\section{VOCES A FAVOR Y EN CONTRA DE LOS DEBERES}

Las críticas sobre los deberes aparecen cuando no se logran encontrar correlaciones significativas con el rendimiento académico (Kohn, 2007a). Algunos autores dicen que esta relación podría depender de la asignatura de que se trate. Por ejemplo, en ciencias sociales o en lectura, podría haber relaciones más favorables que en otras (Walberg, Paschal y Weinstein, 1985). Por otro lado, Skinner, (2004) sostiene que el $55 \%$ de los estudiantes que hacen las tareas tienen mejores resultados comparados con aquellos que no las hacen.

El mayor reclamo en contra del deber es el hecho que la mayoría de las veces no existe retroalimentación (Corno, 2000) o no existen consignas claras y el docente manda la tarea sólo para cumplir con la expectativa tanto propia como de los padres (Kohn, 2007b).

Los deberes pueden atentar también con la agenda familiar, al disminuir el tiempo libre que las familias tienen para compartir (Jackson 2007; Kohn, 2007b; Marzano y Pickering, 2007;
Ohanian, 2004, Xu, 2006).

De todos modos, los deberes pueden ser una oportunidad de unir el trabajo de la casa con el de la escuela, especialmente, cuando se integran tareas cotidianas a los mismos (Corno, 2000). Para niños con dificultades, poco estimulados y de ámbitos desfavorecidos, la tarea domiciliaria puede ser un medio de lograr una mayor nivelación con sus compañeros más aventajados (Jackson, 2007).

Es un hecho que los deberes y la forma de concebirlos y vivirlos, tiene que ver con los niveles de escolarización, con la forma en que son planteados, y con el hecho de si los objetivos se relacionan con las exigencias del nivel (Cooper y Russell, 2002; Corno, 2000; Skinner 2004). Deslandes (2009a) sostiene que las tareas pueden generar tensiones entre los niños y sus padres, siendo una experiencia problemática en el desarrollo de la autonomía, especialmente para aquellos estudiantes con dificultades de aprendizaje y en el desarrollo.

Las creencias de padres y maestros acerca del aprendizaje influyen en las creencias de los alumnos acerca de lo que significa y se debe hacer para obtener el éxito liceal. A su vez, también influye en el esfuerzo que invierten los alumnos en la tarea y en definitiva en los logros obtenidos. Por ejemplo: los efectos de las bajas expectativas comunicadas subliminalmente por la maestra, resultan en la obtención de menores logros y menores habilidades de autorregulación. (Bempechat, 2004).

Otro aspecto que se destaca en las investigaciones realizadas en Canadá por Deslandes, (2009 a) es que la percepción de los padres es poco similar a la de los hijos, en lo que concierne a la proporción de deberes completados y a la percepción sobre la utilidad de las tareas domiciliarias. Las discrepancias y apreciaciones que realizan las distintas poblaciones indican que, existen distintos motivos que estarían participando en la concreción de las tareas. Entre ellos, una estructura diferencial de recompensas y presiones, tanto institucionales como familiares.

En la mayoría de los casos las tareas responden a una estructura de incentivos externa: los deberes son el medio para obtener las calificaciones necesarias que posibilitan el acenso académico en las instituciones. El punto es, según Goupil (1997), lograr que los deberes respondan a incentivos o motivaciones intrínsecas vinculadas con el aprendizaje. 


\section{LA PARTICIPACIÓN DE LOS PADRES EN LA TAREA DOMICILIARIA}

En general, existe acuerdo en el sentido de que los padres deben jugar un rol fundamental en la tarea domiciliaria. Sin embargo, al examinar la literatura e investigaciones, no queda claro cómo se define este rol.

En el mundo se han realizado distintos estudios acerca del tema, uno de los más relevantes fue el realizado por Hoover - Dempsey y Sandler (1995), Hoover Dempsey et al , 2001 (en Deslandes 2009a), retomado luego por Walker et al (2005) a través de una revisión bibliográfica acerca de la participación de los padres en las tareas domiciliarias. A partir de esta recopilación, se puede señalar que las prácticas de los padres para involucrarse en las tareas domiciliaras se presentan en distintas formas: desde establecer estructuras comportamentales para la realización de la tarea hasta enseñar a sus hijos para que desarrollen estrategias de aprendizaje.

Según estos autores, el involucramiento parental en las tareas domiciliarias, influye en el éxito académico de los estudiantes, debido a que se fomentan las atribuciones de logro, por ejemplo: auto percepción de competencias y habilidades de auto regulación.

Por otro lado, la participación parental pertenece a un amplio rango de actividades cognitivas y afectivas. La interacción paterno - filial enmarcada en el acto de realizar los deberes es compleja. Ella incluye distintas conductas parentales, entre ellas, la preparación del ambiente físico, el control del tiempo, la atención y la motivación para evitar posibles distracciones.

Por su parte, las expectativas de la escuela en relación con las funciones parentales de apoyo a menudo se traducen en actividades tales como: estimular la lectura, poner límites en el tiempo dedicado a las "nuevas pantallas" (televisión, computadora, videojuegos), mostrar interés por las actividades escolares, realizar la supervisión de la tarea diaria, firmar los registros de terminación de la tarea, preocuparse para que los niños cuenten con los materiales necesarios.

En la medida en que no todas las familias tengan el capital cultural, económico y social para apoyar el aprendizaje escolar en el hogar, la tarea puede tener efectos perversos. Este aspecto surge si consideramos la responsabilidad que descarga la escuela en la familia mediante las tareas asignadas. Este hecho puede aumentar la desigualdad de resultados educativos, e incrementar el estrés y la angustia en padres y niños de familias con dificultades.

Existen en la actualidad un creciente grupo de investigaciones (Jeynes 2003, 2007 y Pomerantz et al. 2007 en Deslandes, 2009a) que buscan identificar constructos que puedan explicar la influencia de la participación parental en los logros estudiantiles. Estas líneas de investigación se basan en la utilización de mecanismos psicológicos de aprendizaje, focalizándose en la relación entre los efectos del involucramiento parental y los procesos cognitivos. Por ejemplo, las variables afectivas del apoyo parental han sido identificadas como factores de importante influencia en los logros académicos de los estudiantes.

Hoover-Dempsey et al (2001, en Deslandes 2009 a) afirman que las conductas más generales de la participación parental -como es la expresión de expectativas y aspiraciones por parte de los padres acerca de los aprendizajes de los estudiantes- tiene mayor influencia en los logros académicos de sus hijos que las actividades específicas, como la supervisión de los deberes y las conversaciones entre padres e hijos acerca de los deberes y el trabajo escolar.

Aun así, las investigaciones no han determinado cuánto influye en los logros académicos la participación de los padres en las tareas escolares. Esto, porque el problema reside un paso antes, pues aún no se ha determinado claramente cuál es la relación entre los logros escolares y las tareas domiciliarias.

Autores como Pomerantz, Nyg \& Wang (en Deslandes 2009 a) han encontrado en sus estudios que la expresión de sentimientos positivos por parte de los padres mientras que sus hijos realizan los deberes motiva a los estudiantes a esforzarse más en sus tareas escolares.

Por su parte Deslandes, (2009b), planteó en su estudio realizado en Canadá que las funciones de sostén y apoyo afectivo de los padres forman parte del rol paterno y por lo tanto, el propio rol motiva a los padres a participar en las tareas de sus hijos. Aunque la participación varíe en forma y calidad, los padres en general consideran esta tarea como parte de sus responsabilidades. Lo que ha quedado claro es que la variable relevante en la dimensión "tipo de ayuda" o "tipo de participación parental" (Deslandes 2009 a) es el sostén afectivo de 
los padres y no el conocimiento especifico de contenidos.

Retomando las investigaciones ya mencionadas (Hoover-Dempsey et al, 2001; Jeynes 2003, 2007; Pomerantz et al, 2007 en Deslandes 2009 a) se observa que los logros de los estudiantes en última instancia, dependen de variables que se encuentran a menudo fuera de la influencia de los padres.

En general, las líneas teóricas acerca del tema suelen tomar dos enfoques, reportados por Deslandes (2009 a): uno de ellos asume la existencia de una ruta directa y está representado por autores como Brody et al. 1999; Fan and Chen 2001 y Jeynes 2007, que dan cuenta de una vinculación entre el involucramiento parental en los deberes y los resultados de los alumnos. En este sentido, utiliza como indicadores predictivos de resultados las creencias y conductas parentales.

El segundo enfoque mencionado también por Deslandes (2009 a) recoge aportes de autores como Ibañez et al, 2004; Reynolds 1992; Stenberg et al. 1992; Voelkl 1993, asume la existencia de una ruta indirecta entre la participación de los padres y los logros de sus hijos. Este camino analiza la percepción que tienen los estudiantes del apoyo de sus padres; posteriormente esa información es contrastada con los resultados académicos alcanzados por los estudiantes.

De este modo, la relación entre la participación parental y los resultados de los alumnos debe entenderse como una interacción entre la calidad de la participación parental (acciones parentales de andamiaje afectivo y cognitivo), las características del aprendizaje y el nivel de desarrollo del estudiante. (Deslandes, 2009 a).

\section{LA INVESTIGACIÓN EN URUGUAY}

En el 2008 se comenzó un estudio dirigido por la Dra. González Tornaría de la Facultad de Psicología de la Universidad Católica del Uruguay en colaboración con la Dra. Rollande Deslandes, de la Universidad de Québec à Trois Rivières, acerca de las tareas escolares.

La investigación, que se está desarrollando también en otros países de Europa, Asia y América, se basa en tres objetivos generales. 1. Examinar las creencias y actitudes frente a los deberes y lecciones de los alumnos, sus padres y educadores. 2. Estudiar la relación entre el tiempo consagrado a los deberes y lecciones, la proporción de deberes y lecciones habitualmente completadas, los resultados escolares y las estrategias de autorregulación de los alumnos. 3. Verificar en qué medida ciertas prácticas de participación parental contribuyen al desarrollo de estrategias de autorregulación del alumno a la hora de los deberes y lecciones y a un mejoramiento de los resultados escolares.

La investigación propone dos técnicas para la recopilación de datos: cuestionario o encuesta cerrada y entrevistas semi estructuradas.

En nuestro país, estamos transitando las etapas primeras de la investigación. Hemos traducido, con autorización de la Dra. Deslandes, ambos instrumentos, del original redactado en francés al español y hemos realizado la tarea de retrotraducción de los mismos. Hemos ajustado detalles de giros y vocablos a nuestro contexto, haciendo conocer los instrumentos a docentes y padres vinculados a los contextos en los que serían administrados luego, tal cual se sugiere hacer en los procesos de adaptación de instrumentos. Es esta versión revisada por nativos, la que finalmente hemos administrado en nuestro contexto, para empezar con el pilotaje de los instrumentos.

Los cuestionarios originales en francés han demostrado tener valores muy satisfactorios en cuanto a análisis de fiabilidad para las escalas de comprensión del papel de los padres (0.88) competencia parental (0.86) invitación a los padres para participar de parte de los profesores $(0,90)$ y la participación de los padres en lo que se refiere a los deberes y lecciones en la casa (0.88). Similares índices se han alcanzado en su versión traducida al portugués (Silva, Rousseau y Deslandes, 2009).

Para cumplir con la primera etapa de investigación en Uruguay y pilotear los instrumentos, se han levantado datos en dos instituciones educativas - L1 y L2- cuyas poblaciones pertenecen a estratos sociales diferentes. De acuerdo al "índice de posiciones barriales" (Aguiar- Cardeillac-Chouhy, 2008) la población que asiste a L1 pertenece a un barrio cuya población presenta marcados indicadores de nivel sociocultural bajo. El mismo índice categoriza a la población del L2 como perteneciente a un estrato alto $y$ medio alto.

Se han utilizado los instrumentos de la investigación original dedicados a los alumnos de secundaria. Por lo tanto, se los ha administrado a alumnos de $1^{\circ}, 2^{\circ}$ y $3^{\circ}$ año de liceo, sus padres 
y los docentes de la institución correspondientes a esos grados. A ellos se accedió previa autorización de las direcciones de los colegios y consentimiento de los participantes adultos.

Estos cuestionarios aplicados a padres, alumnos y docentes cuentan con 96, 141 y 71 ítems, respectivamente. En todos los casos los indicadores se valoran según una escala Lickert que va de 1 a 5 puntos. De igual manera que en los instrumentos originales, los ítems están agrupados en dimensiones que responden a distintos constructos teóricos desarrollados por especialistas en el tema de deberes y tareas domiciliarias ya mencionados en la revisión de la literatura internacional (Cooper et al. 2002; Xu, 2006; Hoover-Dempsey et al, 2001; Walker et al, 2005).

Los cuestionarios fueron aplicados a 97 alumnos, 31 docentes y 155 padres en la institución de contexto bajo (L1) y a 17 profesores, 153 padres, 324 alumnos de la institución de contexto alto (L2). La entrevista, por su parte, fue administrada a 6 alumnos, 8 docentes y 13 padres del L1 y a 18 alumnos, 10 docentes y 24 padres del L2.

Actualmente se está iniciando la etapa de análisis de fiabilidad y validez de los instrumentos a partir de los datos obtenidos en estas dos instituciones, tal cual se ha hecho en su momento con su original en francés y con la traducción realizada en otros idiomas, como el portugués. Queda entonces pendiente el reporte de estos estudios para comunicaciones posteriores.

\section{ALGUNAS PRIMERAS REFLEXIONES}

En este primer pasaje de cuestionarios, podemos adelantar, con la prudencia a la que estamos obligados al estar recién transitando la etapa de pilotaje de los instrumentos, que la población más motivada con las tareas domiciliarias es la de los docentes.

Esta opinión podría ser resultado de la formación y del "deber ser" de los docentes, en el cual mandar deberes es lo que ellos esperan de sí mismos y lo que sienten que se espera que ellos hagan. Esto aún a costa de sentirse sobrecargados de tareas, haciéndoseles difícil el planteamiento y la corrección de las mismas, tal cual quedó expresado en las entrevistas. En este sentido, esta primera valoración se acerca a lo reportado en la literatura, en las que las investigaciones plantean que la apreciación de los padres sobre los deberes, es muy similar a la de los niños y menos positiva en general que la de los docentes (Deslandes, 2009 b). Las variables socioeconómicas de las dos instituciones participantes, no parecerían afectar esta opinión de los docentes convirtiéndose así en una constante a la cual adhieren la mayoría de ellos.

Otro concepto que ha mostrado puntos de interesantes de discusión desde nuestras primeras incursiones en el tema, es el concepto de ayuda parental en los deberes. El concepto, tal cual reportamos en el estudio de la literatura, aparece con muchos matices: desde la colaboración concreta con el objetivo planteado con la tarea, hasta la creación de buenas condiciones para que el protagonista de la misma, el alumno, pueda realizarla. Esta dificultad de definir el concepto ayuda y lograr acuerdos, se insinuaría especialmente en las poblaciones de padres y estudiantes, otra vez, acercándonos a las tendencias internacionales sobre el tema (Goupil, 1997). Este punto nos lleva a tener en cuenta lo que Hoover Dempsey y Sandler (1997) dicen sobre la influencia que tiene en la participación parental la percepción que tienen los padres sobre lo que sus hijos y sus docentes quieren y esperan de ellos. Este tema nos compromete, para etapas posteriores de la investigación, a trabajar con las diferentes poblaciones, lo que se entiende por ayuda, ya que ahí pueden radicar los principales puntos de desencuentro sobre el tema de los deberes entre todas las partes.

En este breve recorrido de la investigación internacional, así como en los primeros estudios realizados a nivel local sobre el tema con otros diseños de investigación, (Ayala, 2008; Barrera, 2008) se puede apreciar que la concepción y vivencia de los deberes no sólo afecta las relaciones familiares, sino que además puede ir desgastando la relación que la familia y los niños tengan con la escuela.

Por ello, ha sido evaluado en la literatura como beneficioso, el llegar a acuerdos entre padres y docentes, al inicio de cada año, donde quede claro qué esperan los unos de los otros respecto de este tema, antes que puedan aparecer dificultades al respecto. Ambas comunidades, escuela y familia, realizan actividades diferentes y muchas veces complementarias. Los deberes pueden ser un buen motivo para promover la interacción entre ambas, ya que las familias cuentan con los llamados "fondos de conocimientos" que no deberían ser ignora- 
dos, y que pueden ser especialmente tenidos en cuenta en las tareas escolares, llegando a mejorar el desempeño de los estudiantes (Lacasa y Martín, 1996).

\section{BIBLIOGRAFÍA}

Aguiar, Cardeillac, Chouhy (2008). Posiciones Geográficas de Montevideo- Posiciones Sociales en Montevideo. Facultad de Ciencias Sociales. Universidad de la República.

Ayala, M. E. (2008). Los deberes escolares como mediadores entre escuela y familia. Memoria Final. Postgrado en Psicología Educacional. Universidad Católica del Uruguay. Tutora: Prof. María del Luján González Tornaría.

Barrera, P. (2008). Los deberes y tareas para casa: exploración sobre los objetivos para los que son enviados y su cumplimiento. Memoria Final. Postgrado en Psicología Educacional. Universidad Católica del Uruguay. Tutora: Prof. María del Luján González Tornaría.

Bembenutty, H. (2006). Parent involvement homework and self regulation. www.findarticles.com/p/article/mi hb3325/is_4_10/ai_n29328206/?tag=content;coll Consultado agosto, 2009.

Bempechat, J. (2004). The Motivational Benefits of Homework: A Social-Cognitive Perspective. Theory into practice, 43 (3), 189-196.

Butler, D. L. (1999) Board Journal: National School Board Association. Vol. 194, pp 32-33.

Corno, L. (2000). Looking at homework differently. Elementary School Journal, 100, (5), 529.

Cooper, H. (2003). Homework tips for parents. Education Publication Centre, U.S. Department of Education.

Cooper, H., Lindsay, J.J., y Nye, B. (2000): Homework in the home: How student, family and parenting-style differences relate to the homework process. Contemporary Educational Psychology 25, 464-487.

Cooper, H. G. R. y Russell, M. (2002). A teacher's Guide to Homework Tips for Parents: Talking points for Presenters to use with transparencies. Retrieved.form.?

Deslandes, R. (2009 a). Internacional Perspectives on Student Outcomes and Homework. Family - school - community partnerships. Editado por Rollande Deslandes. Routledge: Contexts of learning.

Deslandes, R. (2009b): Conferencia Deberes y lecciones en Québec: resultados de un estudio transversal y longitudinal realizado en el nivel de primaria. Seminario Los deberes escolares en el marco de la relación familia-centro educativo. Facultad de Psicología. Universidad Católica del Uruguay. 12-13 de mayo.

Epstein, J.L (1988): How do we improve programs for parent involvement? Educational Leadership, 66, 58-59.

González Tornaría, M.L. (2006). La necesidad de colaboración de la relación escuela -familia. Revisión de la formación docente en la temática a la luz de la experiencia canadiense. Montevideo: Centro de Estudios Canadienses Canadá-Uruguay.
Goupil, G. (1997). Communications et relations entre l'école et la famille. Montreal-Toronto: Chenelière/Mc Graw -Hill.

Hoover Dempsey, K. V y Sandler, H. M. (1995): Parental Involvement in Children's Education: why does it make a difference? Teachers College Record, 97, (2) 310-331.

Hoover Dempsey, K.V. y Sandler, H.M. (1997): Why do parents become involved in their children's education? Review of Educational Research, 61 (1), 3-42.

Hoover-Dempsey, K.V; Battiato, A.C.; Walter, J.M.T.; Reed, R.P.; De Jong, J.M. y Jones, K.P. (2001). Parental involvement in homework. Educational Psychologist 36 (3), 195-2009.

Jackson, B. (2007). Homework inoculation and the limits of research. Phi Delta Kappan, 89 (1), 55-59.

Kohn, A. (2007a). Baetting Myths: A Q\&A with autor Alfie Kohn, American School Board Journal (194), 32-33.

Kohn, A. (2007b) Rethinking Homework. Principal, 86 (3), 35-38.

Lacasa, P. y Martín, B. (1996): Los deberes: ¿un puente entre la escuela y el hogar? Cultura y Educación, 4, 45-62.

Marzano, R. J., y Pickering, D.J. (2007). The Case For and Against Homework. Educational Leadership, 64 (6), 74-79.

Ministère de l'Education du Québec (2003). Programme d'aide aux devoirs. http://mels.gouv.qc.ca/lancement/prog_devoirs_ecoles/feuillet_aideauxdevoirs_fpdf. Consultado octubre, 2005.

Ohanian, S. (2004). The Homerwork Revolution. Paths of Learning (21), 27-30.

Rosario P, et al. (2006). Escuela- Familia ¿Es posible una relación reciproca y positiva? Papeles del Psicólogo, 27 (3), 171-179.

Silva, N., Rousseau, M. y Deslandes, R. (2009): Validaçao de instrumento de medida acerca da implicaçao dos pais no acompahamento da realizaçao das liçoes de casa de seus filhos. Revista Acoalfaplp: Acolhando a Alfabetizaçao nos paises de Lingua Portuguesa. Sao Paulo, ano 3, No.6. Disponible en: http:// www.acoalfaplp.net Publicado en marzo, 2009.

Skinner, D. (2004). The homework wars. Public Interest (154), 49-60.

Walberg, H.J., Paschal, R.A., y Weinstein, T. (1985). Homework's Powerful Effects on Learning. Educational Leadership, 42 (7), 76.

Walker, .M.T., Wilkins, A. S., Dallaire, J.R., Sandler, H.M. y Hoover-Dempsey, K. (2005): Parent Involvement: Model revision through scale development. The elementary school journal, 106 (2) 85-104.

Xu, J. (2006): Gender and homework Management Reported by High School Students Educational Psychology, 26 (1), 73-91.

$\mathrm{Xu}, \mathrm{J}$. (2009). Homework management reported by secondary school students: a multilevel analysis. En Deslandes, R. (2009a). Internacional Perspectives on Student Outcomes and Homework. Family - school - community partnerships. Editado por Rollande Deslandes. Routledge:Contexts of learning (110-128). 